\title{
Sequential quadrature methods for RDO
}

\author{
Daniele Peri ${ }^{1 *}$ \\ ${ }^{1}$ CNR-IAC - National Research Council - Istituto per le Applicazioni del Calcolo \\ "Mauro Picone", Rome, Italy \\ *Email address for correspondence: d.peri@iac.cnr.it \\ Communicated by Giorgio Fotia \\ Received on November 11, 2014. Accepted on October 12, 2015.
}

\begin{abstract}
This paper presents a comparative study between a large number of different existing sequential quadrature schemes suitable for Robust Design Optimization (RDO), with the inclusion of two partly original approaches. Efficiency of the different integration strategies is evaluated in terms of accuracy and computational effort: main goal of this paper is the identification of an integration strategy able to provide the integral value with a prescribed accuracy using a limited number of function samples. Identification of the different qualities of the various integration schemes is obtained utilizing both algebraic and practical test cases. Differences in the computational effort needed by the different schemes is evidenced, and the implications on their application to practical RDO problems is highlighted.

Keywords: Quadrature schemes, Robust Design Optimization, Industrial Design.

AMS subject classification: $65 \mathrm{~K} 10,65 \mathrm{D} 32,68 \mathrm{U} 07$
\end{abstract}

\section{Introduction}

Robust Design Optimization (RDO) is performed when uncertainties into the optimization cycle are to be included. Classically, design optimization considers one or more objective functions, obtained by computing/evaluating the performances of a system in some prescribed operative conditions. In reality, some of the parameters influencing the performances of the system are not assigned rigorously, or they cannot be controlled: one classical example, in ship design, is represented by the environmental conditions. In RDO, some of the main parameters are regarded as stochastic variables, possibly with a known probability distribution coming from previous experiences or theoretical considerations. The concept of RDO has been firstly introduced by Taguchi in late fifties, and than further developed in $[1,2]$ : this represents a really active research area, with practical applications in the majority of the industrial fields [3-6]. 
In RDO, the value of the objective function, usually computed in a single operative condition, is substituted by the expected value (and/or variance) of a specific quantity characterizing the system over the assumed interval of variation of the stochastic variables, weighted by their probability density function. As a consequence, an integral needs to be computed in order to evaluate the probabilistic quantities associated with the new definition of the optimization problem.

Uncertainty Quantification (UQ) is aimed to this purpose: identify the degree of complexity of the dependence between the objective function and the stochastic variables, quantifying the uncertainties of the objective function subject to stochastic parameters. To do that, one of the main points is the identification of an accurate estimate of the expected value and variance of the objective function. This operation is performed through the evaluation of the integral of the objective function on the stochastic domain

$$
E(X)=\int_{X} f(x) \cdot p(x) d x,
$$

where $X$ is the integration domain, $f(x)$ is the objective function and $p(x)$ is the probability density function, for which holds the property

$$
\int_{X} p(x) d x=1 .
$$

If the expected value $E(X)$ represents the objective function of the optimization problem, the integral (1) needs to be computed in order to provide the value of the objective function, and it will be computed each time the objective function value is required by the optimization algorithm: as a consequence, this operation could be performed a really large amount of times during the optimization process. Consequences may become even more critical if the objective function is provided by a complex numerical simulation tool, since the computational time required by this class of solvers is typically high. The precision of the discrete form of (1) is really important, because the optimization algorithm may discriminate between two different system configurations, and sometime their relative difference is of the same order of magnitude of the accuracy of the evaluation of (1), in particular when local information about the objective function (gradient or Hessian) are required. In literature, quadrature schemes are adopted for the evaluation of (1). The integral is reduced in the form

$$
I=\int_{X} f(x) d x \simeq \sum_{i=1}^{N} f\left(x_{i}\right) w_{i} .
$$




\section{Sequential quadrature methods for RDO}

In other words, a number of punctual values of the objective function are computed in some selected points $x_{i}$, and the integral is obtained by applying a proper weight $w_{i}$ to each computational point. The accuracy of the integration scheme is connected with the number of points adopted for the quadrature formula, so that an appropriate selection is necessary in order to support the optimization algorithm in discriminating between two similar solutions, limiting at the same time the order of the scheme - and the number of necessary sample points. This operation is commonly performed once and for all analyzing the original configuration only: different schemes are tested, changing their order, as soon as the most economic combination is identified. The selected combination will be adopted without any further change during all the optimization process.

Anyway, the modification of the system configuration during the optimization process does not guarantees the conservation of the good features of the integration procedure with respect to the stochastic variables, and a constant verification of the accuracy of (2) is needed in order to guarantee a prescribed level of accuracy along the optimization process. In other words, the position of the integration points is fixed once the order of integration is selected, and their position is not changing if a different integrating function (generated by the change of the system configuration) is considered: position of the integration points may be particularly suitable for the initial configuration of the problem, but this feature may be lost once the system configuration is changing. For this reason, integration scheme is required also to be able to provide an estimate of the accuracy of the integral: this feature can be obtained i.e. by comparing the results obtained rising of one unit the order of integration. Order of integration is further increased until the accuracy of the integral computation is satisfactory. Unfortunately, some of the most popular quadrature schemes do not share the integration point positions when the order of integration is changed, and the computational price of the procedure may become huge. On the contrary, sequential quadrature schemes are able to recycle some of the previously computed sample points when the order of integration is changed, so that they appear to be more suitable to this context. For this reason, they will be considered in this particular application. Since the overall numerical effort is largely dependent upon the selection of the integration scheme, this paper is aimed at the identification of the most economic integration strategy.

In the following sections, a number of sequential quadrature schemes will be presented. Some interpolation and approximation methods, able to provide the exact value of the integral above the interpolating/approximating curve, will be proposed. Then, all the different integration schemes will be tested on a practical ship design exercise, in order to evaluate also the ef- 
fect of the presence of numerical noise: an algebraic reformulation of the ship design problem is adopted for the fast selection of the most promising schemes, and finally the most promising ones are applied to the real ship design problem. As a conclusion, some examples of numerical optimization procedure will be presented and discussed under the perspective of the reduction of the overall computational effort.

\section{Sequential quadrature formulae}

The most simple strategy for the evaluation of an integral is the trapezoidal rule: the integration interval is divided into a number of smaller intervals, and the integral is approximated by the sum of the areas of all the intervals

$$
\int_{a}^{b} f(x) d x \simeq \frac{(b-a) \cdot(f(b)+f(a)}{2} .
$$

The accuracy of the computation is connected with the density of the sub-intervals. This is a very poorly efficient strategy, because a dense and uniform subdivision of the integration interval is required. This approach can be viewed as a local linear interpolation of the function: the speed of convergence of this scheme is, consequently, very slow. A faster convergence is obtained once the function is approximated by a series of orthogonal functions, whose integral is known in closed form. This is the approach shared by a large number of quadrature formulae: the integrand is fitted by a series of polynomials, and the value of the integral (1) is provided in the form (2): position of points and value of weights have been computed once for all. If the function is smooth and regular, a very good approximation can be obtained by using a moderate number of points.

One of the most popular schemes is the Gaussian Quadrature formula. This is commonly adopted for a large number of applications and typically provides a good approximation of the integral also with a moderate degree of the polynomials, that is, using a limited number of samples. A large literature for the convergence studies of different versions of the method is available, in [7-9] to report a few, for continuous and discontinuous functions. Since the integral is obtained without an iterative process, no statistical properties of the integral value are available: as a consequence, if we need to check if convergence of the integration procedure has been achieved, in cases where the exact value of the integral is unknown, the only possibility is to evaluate the integral by using two different orders and then comparing the results. Unfortunately, the position of the Gauss point may be completely different if we pass from 2 to 3 integration points and so on: as a 


\section{Sequential quadrature methods for RDO}

consequence, if we want to check the convergence of the value of the integral by comparing two successive values of the discrete estimate, typically we cannot recycle the previously evaluated values of the objective function. This strategy may become really expensive if the objective function integration requires a large number of integration points: the evaluation of the objective function could be repeated thousands of times during the solution of an optimization problem, and each time the integral computation is involved. To give an example, 54 evaluations of the objective function are needed to cover up the range from order 2 to order 10, so that the overall optimization time is increased by a factor 54 with respect to a standard optimization process where the objective function is computed by using a single value.

A sub-class of quadrature formulae is defined as "recursive" (or sequential) because some of the integration points are shared between the different orders of integration. In other words, if we compute the integral by adopting a 2 point scheme and than we want to evaluate the same integral by using a 3 points scheme, we do not need to add 3 new points, while some of the previous 2 ( 1 or 2 , depending from the scheme) have been already computed previously.

The accuracy of the various formulae is connected with the ability of the base functions to interpolate the candidate function, so that the shape of the function may result more suitable for a specific quadrature law, or a set of orthogonal functions may result more flexible than another. As a consequence, a large number of algebraic functions should be adopted in order to derive some generic conclusions. In the present study, our aim is not to identify the overall best integration scheme, but to compare the performances of different schemes in terms of number of integration points in the spite of a sequential approach, identifying the most appropriate and efficient formula to be adopted for some practical and specific applications.

The QUADRULE package ${ }^{\mathrm{a}}$ provides a number of different quadrature rules, already implemented: it has been adopted to this purpose, testing all the different sequential quadrature rules from this package on specific applications. In the paper, the nomenclature adopted by the authors of the package is reported, and we refer to the documentation for more details.

\section{Interpolation and approximation schemes}

The aforementioned quadrature schemes share the basic principle of interpolating a curve by a series of orthogonal functions, whose integral is analytically known. As a consequence, the integral of the curve is obtained

\footnotetext{
${ }^{a} \mathrm{http}$ ://people.sc.fsu.edu/ jburkardt/f_src/quadrule/quadrule.html
} 
by the sum of the integrals of the components. A different approach could be the application of standard interpolation/approximation techniques, selecting those methods able to provide the value of the integral of the limiting curve in closed form. In this paper, we selected one approximation and one interpolation method to compare with standard quadrature rules. As an approximation method, Bézier curves are tightened to this problem: they are highly flexible and adaptable curves, able to provide a precise fit with a prescribed number of points (training points) if the number of control points is large enough. Bézier curves preserve regularity even if the number of control points is high. Furthermore, Bézier curves have an analytical expression of the area included under the curve: by the way, the integral is represented by the sum of the vertical coordinate of the control points [10]. The identification of the Bézier curve fitting a prescribed dataset can be obtained by solving a minimization problem where the objective function if the sum of the square of the distance between the data points and the corresponding point on the Bézier curve.

As interpolation method, we are here adopting cubic splines. Also in this case, the exact value of the integral of the limit curve is available. Cubic splines are more sensible to the effects of numerical noise possibly introduced by the simulations: as a consequence, an uneven spacing of the training points may cause instabilities. On the other hand, they do not require a complex procedure for the determination of the fitting parameters, as required by Bézier curves, so that they are much easier to apply. Since interpolation is possible for every training set, training points can be distributed in a convenient way for a complete description of the integrating curve, depending on the shape of the curve itself. We have here considered two different alternatives. Firstly, we can progressively increase the number of training points preserving the uniformity of the description of the integration interval, presuming an uniform distribution of the prediction error for the model over the integration interval. In this case, we can talk about "uniform distribution": the Halton distribution [11] belongs to the class of the Uniformly Distributed Sequences (UDS), and it has been adopted to this aim. It is sequential, so that the computation of the $N^{\text {th }}$ point is not influencing the position of the previous points. Halton distribution allows the generation of a sequence of points uniformly distributed along the integration interval.

Another approach for the determination of the integration points can be based on the information about the features of two different fitting models. According to [12], we can use the discrepancies between two different interpolation or approximation methods in order to detect areas in which the information about the objective function are poor. Using this approach, 


\section{Sequential quadrature methods for RDO}

we are assuming that the differences between the estimates provided by two different interpolation/approximation models are also indicative about the prediction error. As a consequence, a new training point will be placed in the region where the difference between the two selected fitting models is larger. The goal is to better distribute the training points, reducing their density in regions where the shape of the target function is potentially already well described.

An example is reported in figure ??, where a multimodal function (the same adopted in the following for testing the integration schemes) is fitted by a cubic spline. In the top part of the figure, points are added preserving uniformity: no further information on the fitting function is utilized, so that the procedure will be the same for every function. On the bottom part of the figure, points are added adaptively, comparing two different interpolation models and checking for regions where predictions show larger discrepancies: the sequence of added points will be different from function to function, typically concentrating new points in the regions where the gradient of the fitting function is larger.

Bézier and cubic spline present a great advantage with respect to the classical quadrature formulae in the spite of a sequential evaluation of the integral: all the previously computed points are useful and utilized. As a consequence, if we compute sequentially the integral of the target function starting from order 2, stopping at order 10, this operation requires 10 training point when Bézier or Spline are applied, while it requires 49 function evaluations with Gaussian Quadrature (not fully sequential) and 29 with Clenshaw-Curtis (sequential).

Different strategies can be adopted as stopping criterion for the integration procedure: we can compare the value of the integral obtained using with $N$ and $N+1$ training points, as commonly adopted for quadrature formulae, or we can compare the values of the integral provided by Bézier and cubic spline, stopping the procedure once the two values are below a prescribed limit. In the following, in order to preserve uniformity, the criteria of the convergence of the computed integral will be adopted.

\subsection{Sequential use of integration schemes}

Scope of this paper is not to develop a new quadrature formula or a variant of an existing one: as a consequence, the strategies available from the aforementioned QUADRULE package are adopted. In the table 1, a comparison in term of objective function evaluations required by the available integration schemes in a sequential usage is reported. First column is reporting the order of the integration scheme, the other columns are reporting 
the number of points effectively required if we are starting from a 3 points scheme and we proceed step by step by increasing the integration order of a single point each time. Differences are due to the different requirement of each quadrature formula in fixing the position of the integration points. Unfortunately, position of the integration points is dictated by the base theory, so that they result ineffective in a sequential use of the scheme, since a large part of the previously computed points are discharged. The data reported in table 1 are independent from the function we are going to integrate: here we are simply checking how many points are recurring during the different degrees of integration for each integration scheme. In the table, the number of function evaluation needed to cover up all the precision levels from 3 up to a 20 point are reported. We can see from table 1 how a fully non-sequential scheme requires 207 evaluations of the integrating function for evaluating all the alternatives between 3 and 20-points scheme. Gauss-Legendre is reusing the central point and the extreme points, so that it saves 18 point: 189 points are required for the investigation of all the same levels. On the other side, the Clenshaw-Curtis requires only 121 evaluations, saving 86 objective function evaluations (42\%). Bézier, as well as Cubic Splines, is using all the available points, so that the order of integration is equal to the number of points (saving $90 \%$ of the computations): they are the most economic, and the most efficient integration schemes under this perspective. The forecoming analyses will tell us if the precision of the computed integral is comparable with the classical quadrature rules.

\begin{tabular}{|l|l|l|l|l|l|l|l|l|l|l|}
\hline Ord. & NS & GA & CC & F1 & F2 & JA & LO & RA & NC & BE \\
\hline 3 & 3 & 3 & 3 & 3 & 3 & 3 & 3 & 3 & 3 & 3 \\
4 & 7 & 7 & 5 & 7 & 7 & 7 & 5 & 5 & 5 & 4 \\
5 & 12 & 11 & 7 & 11 & 11 & 11 & 7 & 10 & 9 & 5 \\
6 & 18 & 17 & 11 & 17 & 17 & 17 & 11 & 16 & 13 & 6 \\
7 & 25 & 23 & 13 & 23 & 21 & 23 & 15 & 23 & 17 & 7 \\
8 & 33 & 31 & 19 & 31 & 27 & 31 & 21 & 31 & 23 & 8 \\
9 & 42 & 39 & 23 & 37 & 31 & 39 & 27 & 40 & 31 & 9 \\
10 & 52 & 49 & 29 & 45 & 41 & 49 & 35 & 49 & 37 & 10 \\
11 & 63 & 59 & 33 & 55 & 45 & 59 & 43 & 60 & 45 & 11 \\
12 & 75 & 71 & 43 & 63 & 57 & 71 & 53 & 72 & 55 & 12 \\
13 & 88 & 83 & 47 & 73 & 63 & 83 & 63 & 85 & 63 & 13 \\
14 & 102 & 97 & 59 & 85 & 71 & 97 & 75 & 98 & 75 & 14 \\
15 & 117 & 111 & 65 & 91 & 79 & 111 & 87 & 112 & 87 & 15 \\
16 & 133 & 125 & 73 & 107 & 95 & 125 & 99 & 126 & 95 & 16 \\
17 & 150 & 139 & 81 & 121 & 101 & 139 & 113 & 142 & 111 & 17 \\
18 & 168 & 157 & 97 & 131 & 119 & 157 & 127 & 156 & 127 & 18 \\
19 & 187 & 169 & 103 & 149 & 125 & 169 & 143 & 173 & 139 & 19 \\
20 & 207 & 189 & 121 & 163 & 137 & 189 & 159 & 189 & 157 & 20 \\
\hline
\end{tabular}

Table 1. Number of points required for each level of accuracy by the different integration schemes. First column is reporting the order of the integration scheme, then the number of points required for each scheme at the reported level. $\mathrm{NS}=$ Non-Sequential, $\mathrm{GA}=$ Gauss-Legendre, $\mathrm{CC}=$ Clenshaw-Curtis, F1 = Fejer (1), F2 = Fejer (2), JA = Jacobi (ElhayKautsky), $\mathrm{LO}=$ Lobatto, $\mathrm{RA}=$ Radau, $\mathrm{NC}=$ Newton-Cotes, $\mathrm{BE}=$ Bézier. Gauss-Legendre and Gauss-Jacobi behave identically. 


\section{Sequential quadrature methods for RDO}

\section{Practical ship design example}

In order to define a practical design exercise for which integration of the objective function is non trivial, an example from ship design has been adopted, so that a catamaran hull and their performances will be the base element for this study. Main characteristics of the selected ship hull are reported in [13]. The choice of a catamaran hull is driven by the observation of a strong effect of wave interference between the two demi-hulls, reflecting on the powering requirements of the catamaran, on the radiated waves and their impact on the shore. In fact, depending on the shape of the hull, the mutual distance of the two demi-hulls and the forward speed, the two wave systems generated by each single demi-hull interacts is a way such that the powering of the ship may experience strong variations: this kind of interference could be positive or negative, in the sense that the wave resistance coefficient of the catamaran (that is, the portion of the total resistance of the hull associated with the production of the wave field) may become higher or lower than the wave resistance coefficient of the isolated single hull, depending on the aforementioned parameters. As a consequence, an accurate design of the catamaran could take advantage of this situation for a specific speed or for a limited speed range, if the ship hull is properly tuned with the wave system: optimization of the hull shape can result in a reduced energy dissipated into the water for a specific speed, limiting the impact of the hull motion on the shore, since a less aggressive wave pattern is impacting the coast. Under this perspective, the consideration of a RDO problem for the optimization of this kind of hulls is straightforward, since we could be interested in extending the speed range for which the wave effects are beneficial. Consequently, the objective function adopted for the RDO of the catamaran is the expected value of the wave resistance coefficient in calm water: it is here computed by using a simple layer potential flow solver in steady flow condition.

Forward speed is here assumed as the uncertain parameter. Here we will adopt a non-dimensional quantity related to the forward speed, that is, the Froude number ${ }^{\mathrm{b}}$. For high Froude numbers (say $F r \geq 0.5$ ) the interference effects is vanishing while it is evident for the lower values (figure 1). For this reason, this study has been concentrated in low-medium Froude number region, $0.25 \leq F r \leq 0.40$, where the variations of the wave resistance is more pronounced, and the complexity of the objective function may evidence the qualities and possibly the difficulties for each investigated integration scheme.

\footnotetext{
${ }^{b} \mathrm{Fr}=\frac{U}{\sqrt{g L}}$, where $\mathrm{U}$ is the forward speed, $\mathrm{L}$ is a reference length and $\mathrm{g}$ is the gravity acceleration
} 


\subsection{Shape of the objective function}

In figure 1 the wave resistance coefficient as a function of the Froude number is represented. On left of figure 1, the comparison of the wave resistance coefficient of the catamaran and its equivalent single hull configuration is shown: here we can observe the oscillations of the wave resistance coefficient in the low Froude regime, while a similar behavior is observed from $\mathrm{Fr}=0.4 \mathrm{on}$. In the central part of figure 1, the sub-region where the integral will be computed is shown. The is regular and smooth, and it appears to be a challenging function to be integrated.

Since the exact value of the integral is unknown, a reference value of the objective function, to be used for judging the convergence qualities of the methods, is not available. The computational cost of a single evaluation of the wave resistance makes the use of a Montecarlo method at full convergence prohibitive. As an alternative, to be adopted for a preliminary selection of the various integration schemes, an interpolation of the wave resistance coefficient has been considered. Being some of the methods based on polynomial interpolation, here we preferred to apply a Fourier expansion of the wave resistance coefficient, in order not to produce an interpolation implicitly favoring on method with respect to another. The Fourier expansion has been truncated at the $5^{\text {th }}$ order, since it has been demonstrated to be sufficient for a good fitting of the original function: in fact, the corresponding coefficients are vanishing from this order on. The effect of the approximation is reported in the right end side of figure $1^{\mathrm{c}}$. The exact value of the integral of the Fourier expansion is analytically known, so that the performances of all the different integration schemes can be evaluated. Observing the results obtained on the Fourier expansion of the wave resistance coefficient, a selection of the best integration schemes will be performed, an the selected schemes will be applied to the real data (coming from the numerical simulation): results may be different due to the presence of numerical noise in the simulation.

\section{Uncertainties Quantification for the wave resistance}

In this section, all the different integration schemes will be tested on an algebraic function, mimic the behavior of the wave resistance coefficient of the selected ship in the speed range in between Froude number 0.25 and 0.4. In particular, the effective value of the wave resistance on the select interval will be assumed as the quantity of interest. After that, a selection of the best performing integration schemes will be applied directly on the

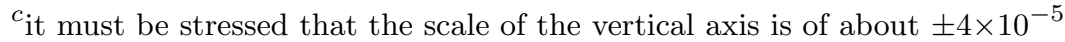




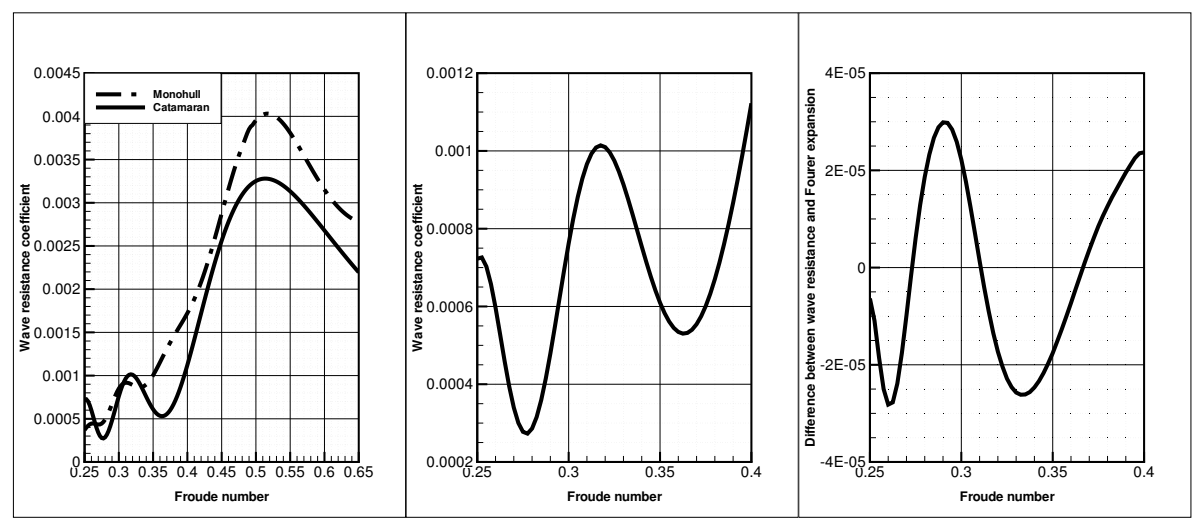

Figure 1. Form left to right: shape of the objective function in a large Froude number range (comparison of the catamaran with the corresponding isolated single hull), zoom of the objective function on the the integration domain and difference between the true objective function and the Fourier expansion.

data coming from the numerical simulation of the steady motion of the ship in calm water.

\subsection{Fourier expansion of the objective function}

Here a Fourier expansion of the wave resistance coefficient is replacing its true value: consequently, the real value of the integral over the assigned interval is analytically known. All the available integration schemes have been applied on this problem, monitoring the value of the integral and the percentage difference between two successive iterations (corresponding to different orders of the scheme). In practical applications, the latter quantity only can be adopted as a stopping criteria for the scheme, being the real value of the integral unknown: once the predicted value of the integral becomes stable from iteration to iteration, we can stop increasing the order of the scheme.

In table 2, the accuracy of the integral computation is reported. Among the 16 different schemes included in the package, some of them are exactly replicating each other: for this reason, they have been excluded from the forecoming computations. By the way, also Jacobi and Legendre produce exactly the same results: they have been reported in the table simply to certify this situation, and Jacobi will be not used in further tests.

Integration schemes reported in table 2 are ranked by the number of evaluations required when the target precision is $0.1 \%$ : this value has been selected because it will be used in the forecoming optimization problem so- 


\section{Peri}

\begin{tabular}{|l|l|l|l|l|}
\hline Scheme & $1 \%$ & $0.1 \%$ & $0.01 \%$ & $0.001 \%$ \\
\hline Spline Uniform & 11 & 12 & 20 & 20 \\
Spline Adaptive & 10 & 15 & 19 & 19 \\
Bézier Uniform & 10 & 16 & 27 & 50 \\
Bézier Adaptive & 10 & 17 & 43 & 108 \\
Radau & 16 & 23 & 40 & 50 \\
Newton-Cotes Closed & 20 & 25 & - & - \\
Lobatto & 21 & 27 & 35 & 43 \\
Clenshaw-Curtis & 19 & 29 & 33 & 47 \\
\hline Gauss-Legendre & 23 & 31 & 39 & 49 \\
Fejer (1) & 31 & 31 & 45 & 87 \\
Jacobi (Elhay-Kautsky) & 23 & 31 & 39 & 49 \\
Legendre (Stroud-Secrest) & 23 & 31 & 39 & 49 \\
Jacobi (Stroud-Secrest) & 23 & 31 & 39 & 49 \\
Legendre (Davis-Rabinowitz) & 23 & 31 & 39 & 49 \\
Legendre (Elhay-Kautsky) & 23 & 31 & 39 & 49 \\
Gegenbauer & 23 & 31 & 39 & 49 \\
Legendre (Golub-Welsch) & 23 & 31 & 39 & 49 \\
\hline Fejer (2) & 27 & 41 & 63 & 79 \\
Newton-Cotes Open & 35 & 47 & - & - \\
Newton-Cotes Half & 27 & 53 & - & - \\
\hline
\end{tabular}

Table 2. Convergence analysis for different quadrature schemes. Under four different convergence criteria, that is, the percentage variation of the numerical value of the integral between two successive iterations, the number of points required at the current iteration is reported.

lution. Furthermore, the resulting number of evaluations is pretty high but still acceptable for practical applications. Schemes with the same outcome have been grouped between two horizontal lines. Clearly, being Spline and Bézier curves purely additive, they are in the top part of the table. Radau is the best performing among the classical integration schemes, while NewtonCotes Open is ranking good, but it is unable to get higher precision limits in within 150 computations due to slow convergence.

A graphical representation of the convergence of the scheme is reported in figure 2. As anticipated, not all the results are plotted, being some of the integration schemes perfectly equivalent. Jacobi and Legendre are reported in the top right part of the figure 2 uniquely with the purpose of certificating this equivalence. All the classical integration schemes suffer from data saturation for order higher than 20 approximatively: over-fitting occurs as well as for polynomials, but the orthogonality of the base functions is probably delaying this phenomenon. Newton-Cotes appears to be not influenced by this phenomenon, and this is connected with the decomposition of the interval: in fact, the whole interval is decomposed into a collection of sub-interval of order 4 , so that the over-fitting problem is not experienced. Spline is providing the faster convergence, and the coherence of the prediction when the number of samples is increasing is evident. On the contrary, Bézier curve requires an optimization procedure for the determination of their coefficients, so that an uncertainty connected with the precision of the base parameters is included: the graph is evidencing this 

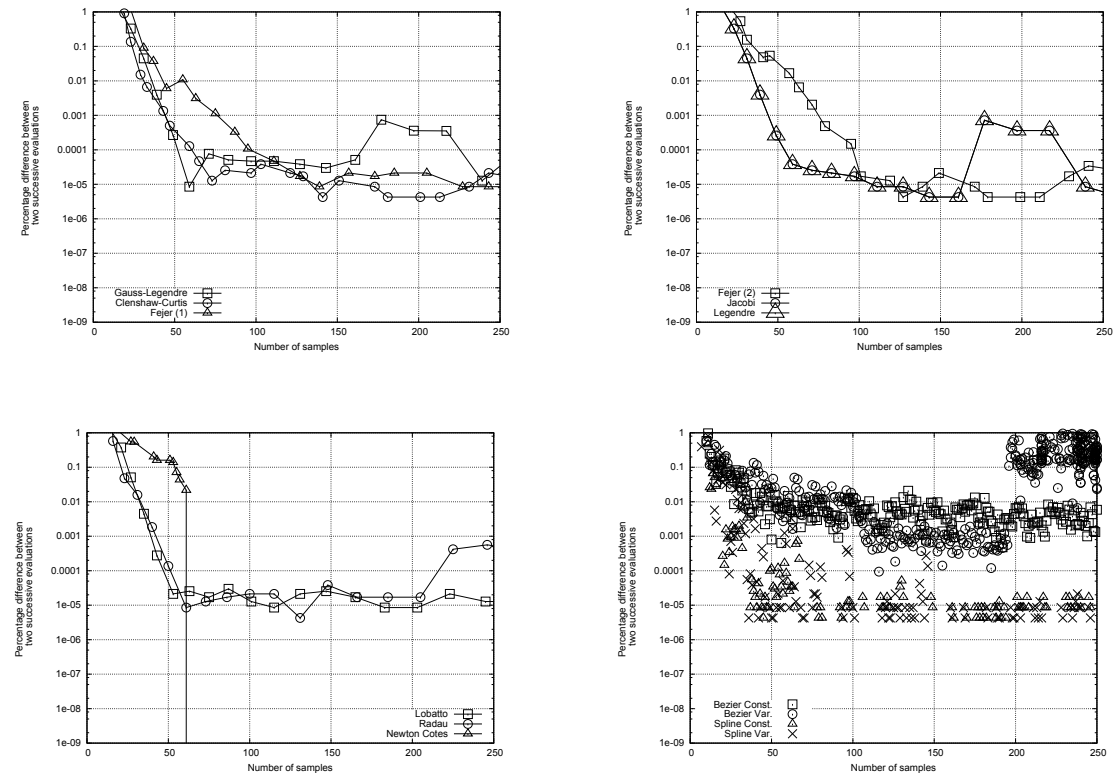

Figure 2. Convergence of the percentage difference between two successive computed values of the integral for the most relevant integration schemes: results obtained on the Fourier expansion of the objective functions. On the horizontal axis, the number of computed points. On the vertical axis, the percentage differences between two successive value of the integral (obtained by raising up the order of the scheme of one unit).

kind of inaccuracies.

Table 3 is similar to table 2, but here the differences between the true value of the integral and the current value provided by the integration schemes are reported. In other terms, while table 3 is presenting elements about the celerity of convergence of each scheme to its asymptotic value, table 2 is providing elements about the exactness of the asymptotic value to witch each scheme is converging to, if convergence is observed. In table 3 , the number of computation associated with an assigned level of precision (with respect to the analytical value of the integral) is reported. Schemes are again ranked by the number of evaluations needed in order to reach a precision limit of $0.1 \%$. Ranking order is pretty different from the one reported in table 3, and this difference is due to two different factors: first, the difference between the asymptotic value and the true value; second, the stability of the asymptotic value. As for the previous data from table 2, the criteria is fulfilled if a certain level is obtained: unfortunately, we have 
no indication about the stability of the precision level. In other words, we cannot state that a good approximation of the true value of the integral has been obtained with stability or occasionally. Keeping in mind this consideration, we observe how Radau quadrature scheme approached the true value rapidly, since a difference smaller than $0.1 \%$ is obtained by using 10 computations only. A comparison with a standard Montecarlo method is reported on the bottom of the table: the application of this approach is not a viable solution in this context.

In figure 3 , the convergence of the integral with respect to its analytical value is reported. Newton-Cotes is presenting the most regular behavior, but also the slowest convergence speed. Both Spline and Bézier are able to get a good value of the integral with a small number of computational points, with accuracy similar to classical integration schemes. They produce very good results when the required accuracy is not so stringent. Bottom left frame of the picture is reporting the fast convergence of Radau and Lobatto schemes, but it is also evidencing some irregularities in their behaviour, unlike Gauss-Legendre and Clenshaw-Curtis (top left frame of the picture).

\begin{tabular}{|l|l|l|l|l|}
\hline Scheme & $1 \%$ & $0.1 \%$ & $0.01 \%$ & $0.001 \%$ \\
\hline Radau & 10 & 10 & 23 & 31 \\
Bézier Adaptative & 8 & 15 & 15 & 70 \\
Lobatto & 11 & 15 & 21 & 27 \\
\hline Gauss-Legendre & 11 & 17 & 23 & 31 \\
Jacobi (Elhay-Kautsky) & 11 & 17 & 23 & 31 \\
Legendre (Stroud-Secrest) & 11 & 17 & 23 & 31 \\
Jacobi (Stroud-Secrest) & 11 & 17 & 23 & 31 \\
Legendre (Davis-Rabinowitz) & 11 & 17 & 23 & 31 \\
Legendre (Elhay-Kautsky) & 11 & 17 & 23 & 31 \\
Gegenbauer & 11 & 17 & 23 & 31 \\
Legendre (Golub-Welsch) & 11 & 17 & 23 & 31 \\
\hline Bézier Uniform & 8 & 18 & 20 & 53 \\
Spline Adaptative & 10 & 18 & 28 & 62 \\
Spline Uniform & 5 & 18 & 33 & 34 \\
Clenshaw-Curtis & 13 & 19 & 23 & 33 \\
Fejer (1) & 7 & 23 & 31 & 63 \\
Newton-Cotes Closed & 13 & 25 & - & - \\
Fejer (2) & 7 & 27 & 45 & 63 \\
Newton-Cotes Open & 15 & 47 & - & - \\
Newton-Cotes Half & 8 & 51 & -- & -- \\
\hline Montecarlo & 64 & 512 & 4098 & - \\
\hline
\end{tabular}

Table 3. Convergence analysis for different quadrature schemes. Using four different convergence criteria, that is, the percentage difference between the computed value and the analytical value of the integral, the number of points required by each quadrature scheme at the current iteration is reported.

There is not a clear indication if the constant step or the variable step is preferable for the interpolation/approximation methods. Uniform sampling is performing better with Splines, while adaptive scheme is preferable in conjunction with Bézier. In general, the uniform distribution provides more stable and regular results: nevertheless, since instabilities are experienced 


\section{Sequential quadrature methods for RDO}
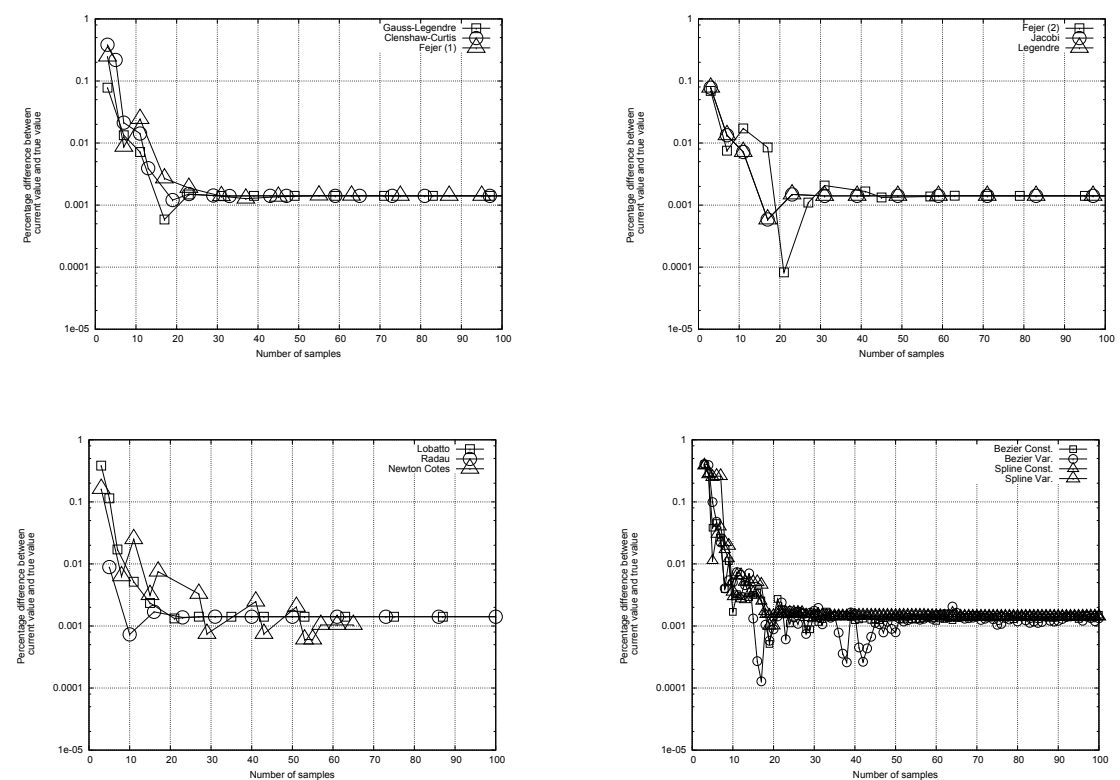

Figure 3. Convergence of the percentage difference between the computed value of the integral and its analytical value for the most relevant integration schemes: results obtained using the Fourier expansion of the wave resistance coefficient. On the horizontal axis, the number of computed points, on the vertical axis, the percentage difference between the computed value and the analytical one.

for a pretty large number of evaluating points, the use of the adaptative approach will be further investigated.

\subsection{Numerical simulation results}

In this section, the mathematical model is directly applied for the determination of the wave resistance coefficient. Since the true value of the integral is not available, we applied a trapezoid method on regular grid (made by 32768 samples), and the final value was $1.023272 \times 10^{-4}$, not far from the analytical value of the Fourier expansion $\left(1.023306 \times 10^{-4}\right)$. Although the effect on a global quantity is not so large, we are going to check the ability of the different integration techniques to deal with the numerical noise typically produced by a simulation code.

Numerical noise is generated by a number of different sources, partly addressed to rounding error. As a consequence, noise is here similar to a random quantity added to the wave resistance coefficient. A quantification 
of the numerical noise is reported in figure 4.
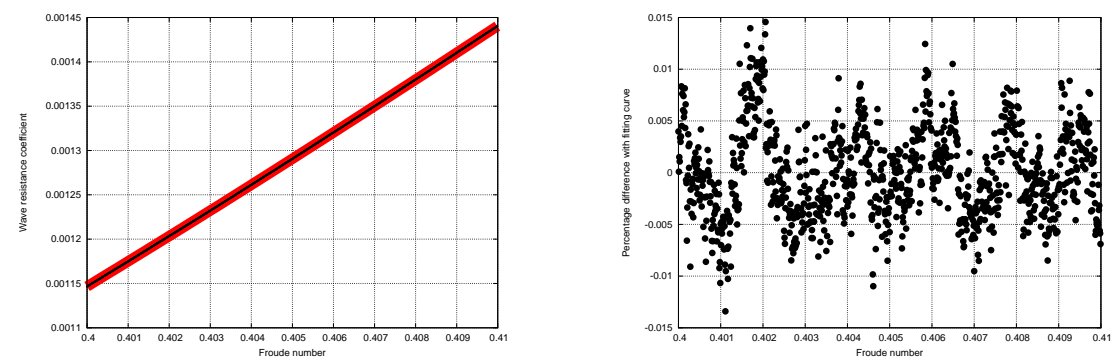

Figure 4. Numerical noise associated with the computation of the wave resistance coefficient for the current application. On left, the wave resistance coefficient as a function of the Froude number: computation on 1001 punctual values is here fitted by a $10^{\text {th }}$ order polynomial. Black dots are representing the samples, thick line is the interpolating polynomial. On left: percentage difference between the polynomial and the true value. The interval has been selected in order to have a nearly linear behaviour of the wave resistance coefficient, so that no further irregularities are introduced.

In figure 5, we can observe the speed of convergence of the selected methods when the real value of the wave resistance coefficient is integrated. Maximum number of computed points has been reduced to 50, in order not to increase the overall computational cost up to unsustainable levels, in the view of an application to optimization. Convergence is slower than the one obtained with the Fourier expansion (reported in figure 2), where we have obtained rapidly convergent values. On the contrary, here classical quadrature rules seems not to be able to reduce convergence more than $10^{-2 \%}$, while Spline and Bézier get the same values as before, preserving a gain of nearly two order of magnitude with respect to classical quadrature rules. In figure 5 the visualization is stopped at 50 integration points, but its behavior is unchanged if we extend the analysis up to 250 integration points.

On the contrary, a substantial equivalence is observed for the precision of the integral. In figure 6 , we are looking at the percentage difference between the real value of the integral and the current value provided by the different integration strategies. All the schemes are stabilizing on a value close to $10^{-1 \%}$ : also in this case, the graph has stopped at 50 integration points, but results are not changing if we increase up to 250 integration points. In this case, the incomplete convergence of the Montecarlo method could play a role. 

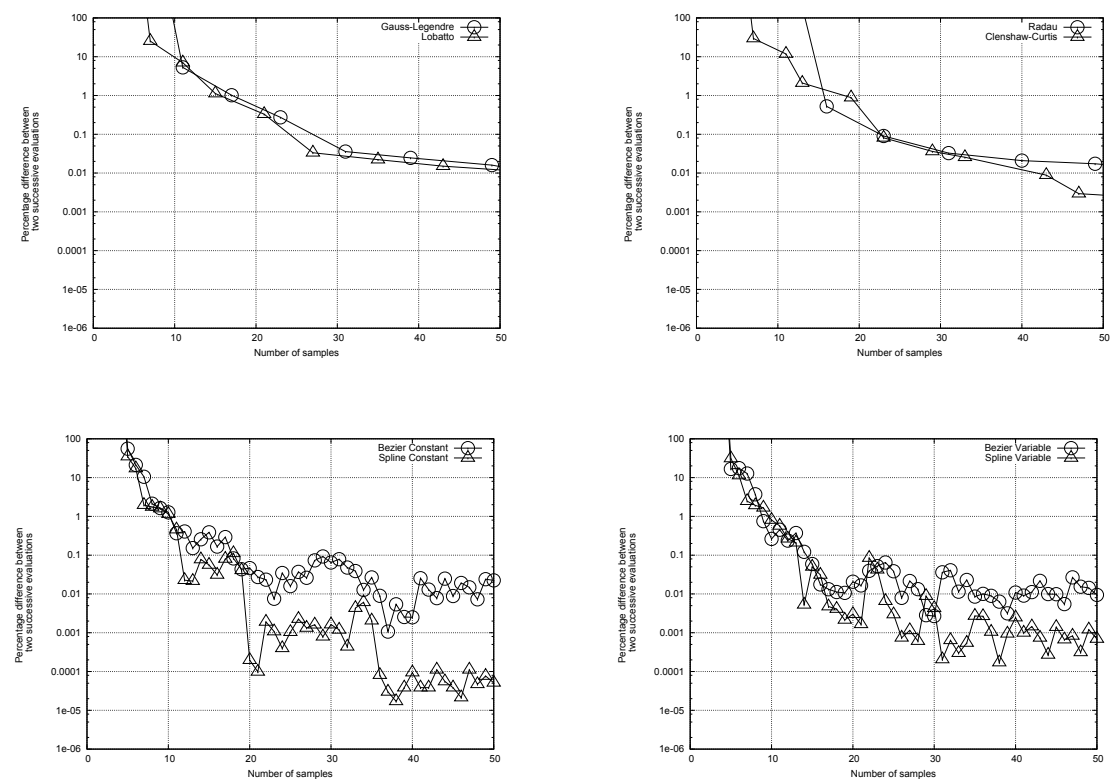

Figure 5. Convergence of the percentage difference between two successive evaluations of the integral for the most relevant integration schemes: results obtained using the simulation tool. On the horizontal axis, the number of computed points. On the vertical axis, the percentage difference between two successive evaluations of the integral.

In conclusion, Spline and Bézier outperforms the other integration schemes if the celerity of convergence of the computed value is adopted as a criteria: a reduced number of integration points could be required if these schemes are applied during the optimization loop, without any loss of precision. Correctness of this sentence has been investigated in the next section.

\section{Optimization test case}

In this section, a complete optimization process has been performed adopting different choices for the integration scheme. As in the last example, the objective function is the expected value of the wave resistance coefficient of the Delft catamaran, computed by mathematical model.

In order to define an optimization problem, a parametric expression of the hull geometry is required. To this aim, parameterization of the hull has been obtained by using an FFD (Free Form Deformation) approach [14,15]. 

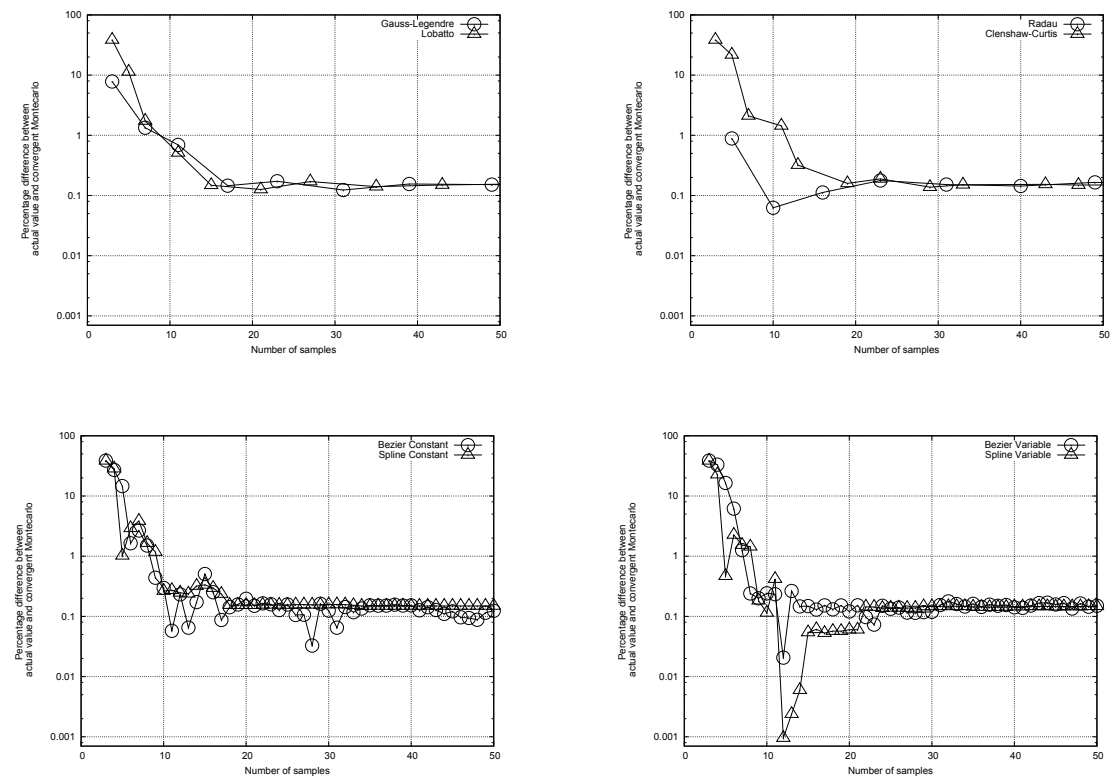

Figure 6. Convergence of the percentage difference between the computed value of the integral and its value provided by a Montecarlo method. Results are obtained by using the true objective functions. On the horizontal axis, the number of computed points. In the vertical axis the difference between the current value and the reference value obtained by Montecarlo simulation.

In this method, the object to be deformed is embedded into a parallelepiped. The parallelepiped is subdivided into regular slices along the three Cartesian axis. A selection of the corners (cross intersection of the dividing lines) are shifted along one or more directions, and the volume included into the parallelepiped is modified according to the movement of the control points. A graphical example is provided in figure 7 .

A box embedding the right side of one demihull is modified by 5 different control points. The demi-hull symmetry is preserved since the left side of the demihull is obtained by mirroring the right side. The optimization process has been repeated changing the integration scheme. Precision limit for the scheme is fixed at $10^{-1 \%}$ : this quantity is not particularly small because we do not want to increase too much the computational effort, due also to the limited available hardware.

Optimization has been performed by using Adaptive and Uniform Splines, Adaptive and Uniform Bézier curves, Gauss-Legendre, Newton- 


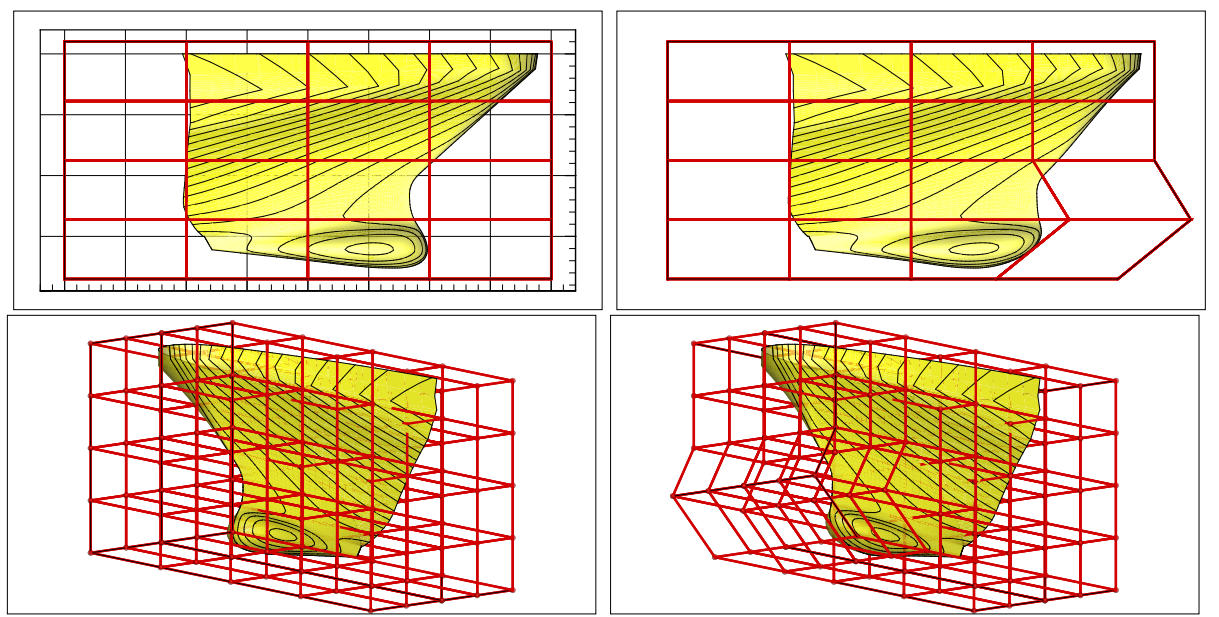

Figure 7. Example of FFD applied to the bow of a surface combatant. On left, the undeformed geometry, on right, the geometry after modification. Only two design variables are shifting the selected control points along the longitudinal axis. Only the lower part of the bow is modified.

Cotes Closed, Lobatto and Radau schemes. Selection has been performed looking at the best ranking obtained from table 2 and 3 . Newton-Cotes has been added due to the good regularity, also if the overall performances are not at the top. Consequently, 8 different optimal configurations have been obtained, one for each integration scheme. A perspective representation of the hull shape before and after the optimization process is reported in figure 8. Deformation of the hull shape is evident. Optimal configuration obtained by using the Radau scheme is reported: the other optimal shapes do not present large differences with respect to the one reported in figure 8 .

Figure 9 is reporting a comparison of the performances of the optimal configurations with respect to the original one, and the differences between the solution provided by adopting a different integration scheme. In the top left graph of figure 9 the improvement of the wave resistance coefficient in the lower range of speeds is evident. On the contrary, we observe a small increase of the wave resistance in the high speed range (Froude number greater than 0.375). This is a classical result of RDO: the average value is minimized, but we have not the guarantee (unless some further constraints are utilized) that the objective function is reduced over the entire investigated range. The other graphs in figure 9 are presenting a substantial equivalence of the optimal solutions provided by the different integration schemes. However, a deeper analysis of the results is revealing some differences between the optimal solutions. 
D. Peri
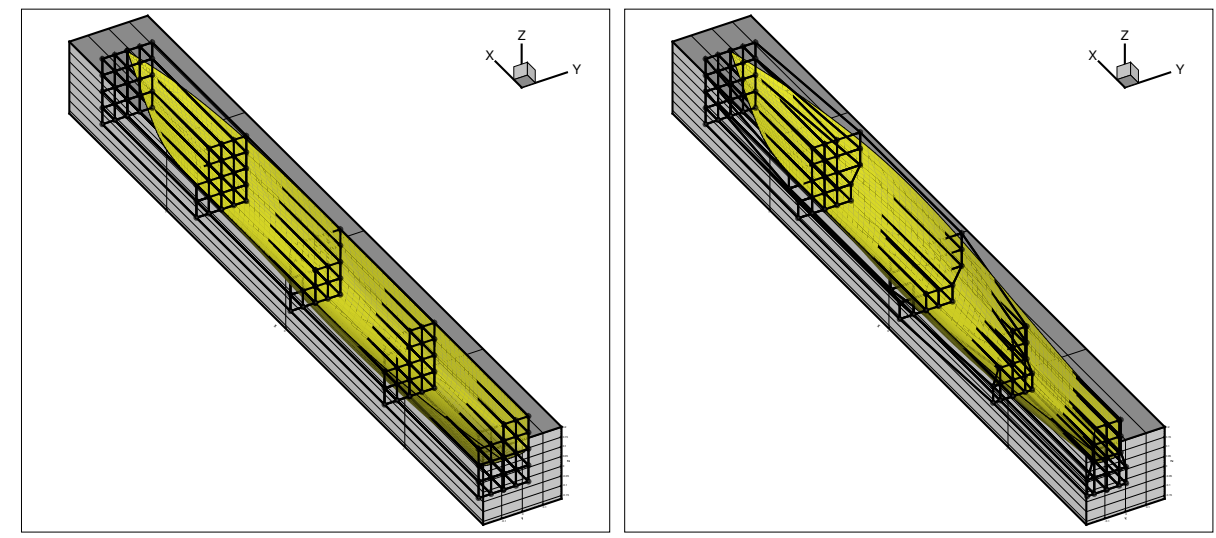

Figure 8. Modification of the Delft catamaran hullform at the end of the optimization process by Radau scheme. Hull geometry and FFD frame are reported. On left, the original geometry, on right, the geometry after modification. Five design variables are shifting along the longitudinal and lateral axis the FFD frame. Some of the corners are linked together, while the central part of the frame is locked in order to preserve continuity on the symmetry plane.
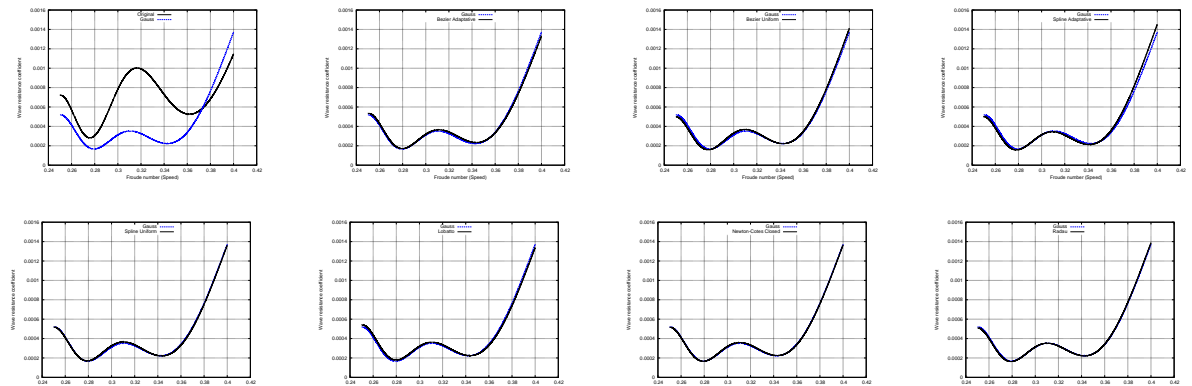

Figure 9. Comparison of the integrand function (wave resistance coefficient) over the full speed range before and after the optimization process. On top left, a comparison between original and optimal geometry produced by using the Gauss-Legendre integration scheme is reported. On the other pictures, a comparison of the results provided by the Gauss-Legendre scheme and all the other alternatives are reported.

In table 4, some significative quantities are reported. First column is indicating the adopted integration scheme. Second and third columns are reporting the estimated value of the integral and its exact value. Exact value is computed by means of a regular sampling of the integration inter- 


\section{Sequential quadrature methods for RDO}

val, using 32768 points, and than applying the simple trapezoidal rule: a convergence study is guaranteeing the precision of the integral up to the fifth digit. Fourth column is reporting the percentage error between the exact value and the estimated one. Estimated value is computed using the indicated quadrature method: order of the method is increased sequentially, and the procedure stops when the percentage difference between two successive evaluations is lower than $0.1 \%$. Quadrature scheme is reliable if the value reported in the fourth column is of the same order of magnitude of the stopping criteria. Under this perspective, all the investigated methods meet the requirement: Gauss-Legendre, Lobatto, Radau and Bézier Adaptive are providing an estimate whose precision is one order of magnitude lower than required, while the error observed for all the other is of the same order of magnitude of the accepted one.

The fifth and sixth columns of table 4 are reporting the optimal value of the objective function: again, both values provided by the quadrature scheme (with approximation and true value) are reported. Seventh column is reporting the percentage difference between the two. Results are showing something different with respect to the previous ones: in fact, some of the quadrature schemes are showing greater differences, well above the adopted precision limit. Bézier and Adaptive Spline are providing differences of one order of magnitude larger than the adopted precision limit, while all the others are confirming the results of the test on the original geometry. This is a confirmation of the idea that the results provided by the quadrature schemes are problem-dependent, and a single test is not a guarantee of the accuracy of the integral computation along the full optimization problem solution.

Last column is reporting the full number of simulations needed for the complete solution of the optimization problem. This is one of the most important elements, since it gives a measure of the time needed for the full optimization problem solution. Gauss-Legendre, Lobatto and Radau schemes are substantially equivalent: if we compare their requirements with the ones of a full sequential scheme, like Bézier and Splines, we observe a reduction of the computational effort of about $30-40 \%$ by the Bézier and about $60 \%$ by the Splines. Among the last ones, Uniform Splines are able to preserve the accuracy, and their results are comparable with the outcome of some of the classical quadrature formulae.

In figure 10 the differences between approximated value and true value are evidenced. In this picture, the cases of the optimal configurations are reported. The Bézier Uniform (BU) scheme is providing the best solution when comparing the approximated value, while this configuration reveals to be the better one once the true value is computed. $\mathrm{BU}$ is also providing 
D. Peri

\begin{tabular}{|l|l|l|l|l|l|l|l|}
\hline Method & \multicolumn{3}{|c|}{ Original } & \multicolumn{3}{c|}{ Optimal } & Points \\
\hline & Quad. Form. & Exact & \% diff. & Quad. Form. & Exact & \% diff. & \\
\hline RA & $1.023003 \mathrm{E}-4$ & $1.023272 \mathrm{E}-4$ & 0.0263 & $6.363469 \mathrm{E}-5$ & $6.363722 \mathrm{E}-5$ & 0.0040 & 29475 \\
\hline LO & $1.023064 \mathrm{E}-4$ & $1.023272 \mathrm{E}-4$ & 0.0203 & $6.366491 \mathrm{E}-5$ & $6.367956 \mathrm{E}-5$ & 0.0230 & 26952 \\
\hline GA & $1.023562 \mathrm{E}-4$ & $1.023272 \mathrm{E}-4$ & 0.0283 & $6.361283 \mathrm{E}-5$ & $6.359716 \mathrm{E}-5$ & 0.0246 & 30828 \\
\hline SU & $1.022296 \mathrm{E}-4$ & $1.023272 \mathrm{E}-4$ & 0.0954 & $6.350983 \mathrm{E}-5$ & $6.360077 \mathrm{E}-5$ & 0.1432 & 11974 \\
\hline \hline NCC & $1.021242 \mathrm{E}-4$ & $1.023272 \mathrm{E}-4$ & 0.1984 & $6.334666 \mathrm{E}-5$ & $6.359032 \mathrm{E}-5$ & 0.3846 & 53458 \\
\hline BA & $1.023114 \mathrm{E}-4$ & $1.023272 \mathrm{E}-4$ & 0.0155 & $6.264679 \mathrm{E}-5$ & $6.373667 \mathrm{E}-5$ & 1.7397 & 22543 \\
\hline SA & $1.020111 \mathrm{E}-4$ & $1.023272 \mathrm{E}-4$ & 0.3089 & $6.318611 \mathrm{E}-5$ & $6.449119 \mathrm{E}-5$ & 2.0654 & 11853 \\
\hline BU & $1.020887 \mathrm{E}-4$ & $1.023272 \mathrm{E}-4$ & 0.2331 & $6.096539 \mathrm{E}-5$ & $6.438658 \mathrm{E}-5$ & 5.6117 & 16862 \\
\hline
\end{tabular}

Table 4. Objective function value for the original and optimal configuration. 8 different optimization problems have been solved, one for each selected quadrature scheme. Precision limit required for the quadrature scheme is $0.1 \%$. The value provided by the integration scheme with the indicated accuracy is compared with the exact value, computed by using trapezium rule (32768 points). BA = Bézier Adaptative, $\mathrm{BU}=$ Bézier Uniform, $\mathrm{GA}=$ Gauss-Legendre, $\mathrm{LO}=$ Lobatto, $\mathrm{NC}=$ Newton-Cotes, RA = Radau, SA = Spline Adaptative, $\mathrm{SU}=$ Spline Uniform. Data are ordered by the percentage differences between approximated and exact value of the objective function for the optimal configuration. Table is split in two parts, isolating the schemes presenting a precision of the integral of the same order of magnitude of the required precision.

the larger difference between predicted and true value of the integral. Best configuration is the one computed by Newton-Cotes (NC) scheme, but the difference with the true value is pretty large. Gauss-Legendre (GA) and Uniform Spline (SU) behave similarly, with a small preference for GA, due to the higher precision.

A last consideration about the variation of the order of the quadrature scheme with the configuration of the system. Checking the history of the solution obtained by using the Radau scheme, objective function has been computed 1001 times: among them, 27 solutions have been computed by using order 7, 140 with order 8 and 834 with order 9. If a fixed order have been adopted, 9 in this case, the full number of calls to the fluid dynamic solver would be 9009 instead of 29475 . The variability of the order of integration is lower for Gauss-Legendre, since only one solution has been computed by a 6 point scheme, and 19 using an 7 order scheme, all the others requiring an 8 point scheme. Here, since an order 8 is sufficient, 8008 calls were needed for the complete solution. Unfortunately, the information about the maximum order of integration is available not prior than the end of the optimization process, so that we cannot fix a priori the order of the scheme. However, we can observe that an adaptive integration scheme by Uniform Splines is potentially increasing the total number of calls to the solver of about $50 \%$, if compared with Gauss-Legendre at constant order, 


\section{Sequential quadrature methods for RDO}

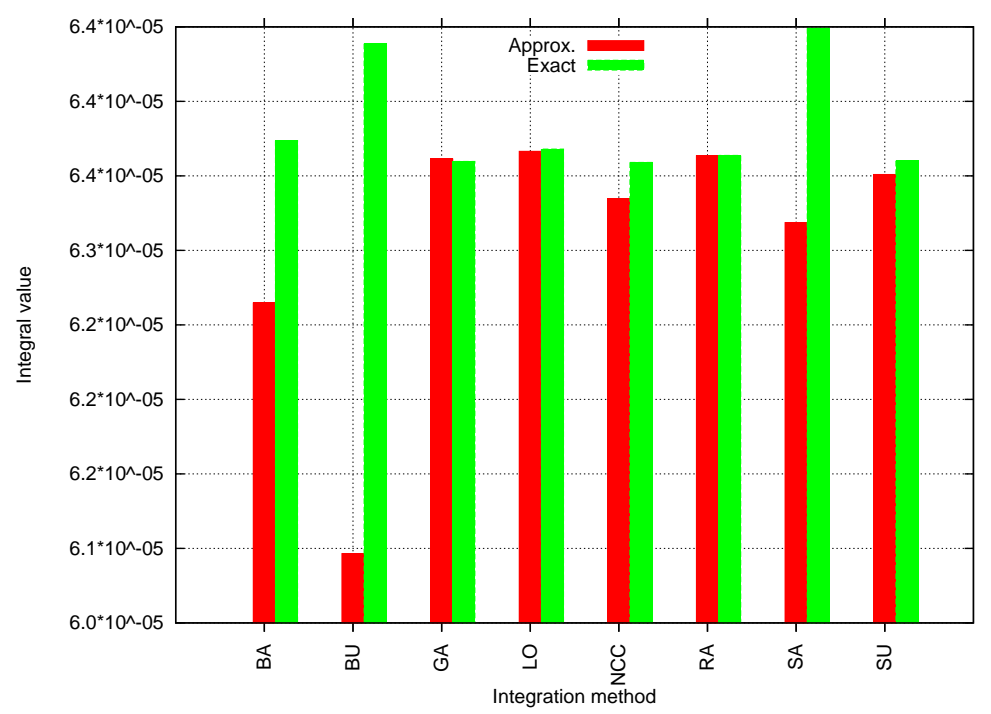

Figure 10. Difference between the approximated value and the true value of the objective function provided by the 8 different integration schemes. Optimal configurations are adopted for the comparison.

but it is also saving $60 \%$ of the calls if compared with a sequential use of the same integration scheme. What is important in the preservation of the accuracy is the effect of each single objective function computation over the evolution of the optimization process: in fact, an erroneous evaluation of the objective function on a single configuration may drive the search away from a promising area, or it can deviate the algorithm toward an uninteresting region. The effects of the inaccuracies in the evaluation of the objective function are particularly evident in the case of the use of a gradient-based optimization algorithm, where the inaccuracy of the gradient approximation is inducing a great loss on the speed of convergence of the algorithm. As a consequence, the highest value of the adopted integration order is to be considered when comparing the price of constant and variable integration order. Table 5 is reporting the order of integration needed for the original and the optimal configuration. In this application, the majority of the integration schemes are using the same order of integration for both the original and the optimal configuration. In few cases, there is an increase of the required order, as for Radau. The only scheme for witch a reduction is experienced is the Adaptive Spline, that is also the most economic scheme, 
but also one of the worst in terms of precision.

\begin{tabular}{|l|l|l|l|l|l|l|l|}
\hline Method & \multicolumn{3}{|c|}{ Original } & \multicolumn{3}{c|}{ Optimal } & Points \\
\hline & $\%$ diff. & Order & Comp & $\%$ diff. & Order & Comp & \\
\hline BA & 0.0155 & 18 & 18 & 1.7397 & 24 & 24 & 22543 \\
\hline BU & 0.2331 & 13 & 13 & 5.6117 & 15 & 15 & 16862 \\
\hline GA & 0.0283 & 8 & 31 & 0.0246 & 8 & 31 & 30828 \\
\hline LO & 0.0203 & 9 & 27 & 0.0230 & 9 & 27 & 26952 \\
\hline NCC & 0.1984 & 11 & 55 & 0.3846 & 11 & 55 & 53458 \\
\hline RA & 0.0263 & 8 & 23 & 0.0040 & 9 & 31 & 29475 \\
\hline SA & 0.3089 & 14 & 14 & 2.0654 & 11 & 11 & 11853 \\
\hline SU & 0.0954 & 12 & 12 & 0.1432 & 12 & 12 & 11974 \\
\hline
\end{tabular}

Table 5. Order of the scheme and solutions needed for a single objective function computation: original and optimal configuration. Precision and full cost of the optimization problem solution are also reported (from table 4). $\mathrm{BA}=$ Bézier Adaptative, BU = Bézier Uniform, GA = Gauss-Legendre, LO $=$ Lobatto, $\mathrm{NC}=$ Newton-Cotes, $\mathrm{RA}=$ Radau, $\mathrm{SA}=$ Spline Adaptative, $\mathrm{SU}=$ Spline Uniform. Alphabetic order.

\section{Conclusions}

Some different additive quadrature schemes have been compared on algebraic function and a practical naval application. A time saving of about $40 \%$ can be obtained by adopting an appropriate quadrature scheme, while the saving becomes close to $60 \%$ if we apply an interpolation/approximation integration scheme. Final results are comparable, so that no preclusion of the usage of Spline and Bézier curves in this context is evidenced. In particular, Uniform Splines provide a level of accuracy similar to the best quadrature schemes, with a strong reduction of the overall computational time. On the contrary, if the order of integration is kept fixed, and there is no room/interest for the qualification of the integral value, the use of the classical integration schemes is still recommended, with some preferences for the Radau scheme.

\section{REFERENCES}

1. G. Taguchi, Introduction to Quality Engineering. Asian Productivity Organization, UNIPUB, 1986.

2. G. Taguchi, S. Chowdhury, and S. Taguchi, Robust Engineering. McGraw-Hill, 2000.

3. D. Goldfarb and G. Iyengar, Robust portfolio selection problems, Mathematics of Operations Research, vol. 1, pp. 1-38, 2003.

4. A. Nilim and L. E. Ghaoui, Algorithms for air traffic flow management under stochastic environments, in Proceedings of the 2004 American 


\section{Sequential quadrature methods for RDO}

Control Conference, vol. 4, pp. 3429-3434, American Automatic Control Council in cooperation with IFAC, 2004.

5. A. Messac and A. Ismail-Yahaya, Multiobjective robust design using physical programming, Structural and Multidisciplinary Optimization, vol. 23, no. 5, pp. 357-371, 2002.

6. M. Diez, D. Peri, G. Fasano, and E. F. Campana, Hydroelastic optimization of a keel fin of a sailing boat: a multidisciplinary robust formulation for ship design, Structural and Multidisciplinary Optimization, vol. 6, no. 4, pp. 613-625, 2012.

7. W. Barrett, Convergence properties of gaussian quadrature formulae, Coputer Journal, vol. 3, no. 4, pp. 272-277, 1961.

8. A. H. Stroud, Gaussian quadrature formulas, in Prentice-Hall series in automatic computation, Prentice-Hall, 1966.

9. P. Rabinowitz, Rates of convergence of gauss, lobatto, and radau integration rules for singular integrands, Mathematics of Computation, vol. 47 , no. 176 , pp. $625-638,1986$.

10. T. W. Sederberg, Computer aided geometric design course notes, tech. rep., Brigham Young University, 2012.

11. J. Halton, Algorithm 247: Radical-inverse quasi-random point sequence, Tech. Rep. 12, Communications of the ACM, 1964.

12. D. Peri, Self-learning metamodels for optimization, Ship Technology Research, vol. 56, pp. 94-108, 2009.

13. R. van't Veer, Experimental results of motions, hydrodynamic coefficients and wave loads on the 372 catamaran model, Tech. Rep. Report 1129, Delft University of Technology, 1998.

14. T. W. Sederberg and S. R. Parry, Free form deformation of solid geometric models, in Proceedings of SIGGRAPH conference (R. A. D.C. Evans, ed.), vol. 20, pp. 151-160, ACM Press, 1986.

15. D. Peri, Conformal free form deformation for the optimisation of complex geometries, Ship Technology Research, vol. 59, pp. 36-41, 2012. 